\title{
Network Performance of Contention Window in VANET
}

\author{
Nasimul Hyder Maruf Bhuyan, Sabrina Alam
}

\begin{abstract}
In this paper, we tried to presented the effect of different contention window $(\mathrm{CW})$ size in a realistic road environment with mobility management and different propagation loss model was implemented in ns-3. We focus on the WAVE module of ns-3. Our contribution is to create a realistic two-lane both way highway road environment surrounding by fields, low-rise commercial buildings, street lamps and road signs for vehicular safety messages are Cooperative Awareness Message (CAM) and Decentralized Event Notification Message (DENM). Medium to low traffic density. On the other section, we describe in detail the simulation setup scenario during the implementation. Finally, we present our simulation results that we obtained from the highway scenario.
\end{abstract}

Index Terms-CAM; Contention Window; DENM; Mobility; NS-3; VANET.

\section{INTRODUCTION}

Research in vehicle-to-vehicle communication or Vehicular Ad Hoc Network (VANET) is one of the major fields of growing interest. The advent and recent development of these concepts foster evolution of the new paradigm is the transportation system, widely known as Intelligent Transport System (ITS). In 1999 US Federal Communications Commission (FCC) allocated $75 \mathrm{MHz}$ (5.850 GHz to $5.925 \mathrm{GHz}$ ) spectrum in the $5.9 \mathrm{GHz}$ band for ITS. The development of ITS and dedicated short-range communications (DSRC) used for the decrease road traumas, improve the road safety, security, efficiency of the transportation systems and improve traffic efficiency for short-to-medium range wireless communications. The road traffic oriented applications can help to avoid the congested roads and fluid the traffic flow. The significant challenges in wireless communication are the hidden station problem, packet collision, channel fading and different types of obstacles. In 2001 ISO Communication Access for Land Mobiles (CALM) which has been worked on IEEE 802.11p and finalized in 2010 [1].

Vehicular Ad-Hoc Network (VANET) communication are established among the vehicles and between the vehicles and Road Side Units (RSUs) for safety and comfort to the passengers [2]. However, for safety application vehicle should be transmitted broadcast packet successfully between 100 milliseconds and 200 milliseconds under normal vehicle density. On the other hand, during the Control Channel Interval (CCI) (50 milliseconds) the broadcast message cannot be transmitted and postponed till next CCIs is re-

Published on February 28, 2018.

N. H. M. Bhuyan is with Blekinge Tekniska Högskola (BTH) and Lund University, Sweden. (e-mail: maruf.feni@gmail.com)

S. Alam is with Electrical and Electronic Engineering, University of Chittagong, Bangladesh. (e-mail: sabrina.apece@cu.ac.bd) transmitted. The Cooperative Adaptive Cruise Control (CACC) uses for the cooperating of vehicle dynamic information (i.e. vehicle position, speed and acceleration) with nearby vehicles for reduce the traffic jams [2]. When a node in VANET cannot get the medium access (e.g. busy channel), then it chooses the interval $[0, \mathrm{CW}]$ of the random bakeoff time and delay the medium. If the packet collision occur and receiver do not get any acknowledgement from the transmitter, then the $\mathrm{CW}$ size is double. However, lack of the beacon acknowledgement in broadcast communication, $\mathrm{CW}$ is never increased for beaconing [2]. For avoiding the maximum access delay time and collision between vehicle-to-vehicle (VTV) broadcast communication the contention window $(\mathrm{CW})$ size have significant role. When the $\mathrm{CW}$ size is small the density of vehicles collision is high and for the large CW size the access delay time is raising [3].

Reference [3] proposed a new Adaptive Dynamic Contention Window (ADCW) scheme, there the size of CW can adjust with vehicles number. This scheme assure that the higher broadcast success rate of vehicles, lower collision rate and the average assess delay, which can save the channel resource and use it for other vehicle services [3]. In broadcast communication the impact of adaptive setting for the $\mathrm{CW}$ was investigated for increasing the traffic efficiency [2]. In [4] the performance of the beacon broadcast reception probability drops below $50 \%$ when $33 \%$ exceeds the channel utilization because the vehicles are sharing the same collision domain and exceeds the threshold level. Two algorithms are designed and implemented using beaconed or beaconless for multi-hop ad hoc broadcast services described in [5]. For the $\mathrm{CW}$ adjustment mechanism and prediction based algorithm by using the sets of attribute of BPR (Bayesian Personalized Ranking) proposed in [6].

The contributions of this paper are as follows:

- Design a realistic highway road scenario in ns-3 and implementation of various $\mathrm{CW}$ size on existing channel model in ns-3.

- Provide analysis both average packet loss ratio and average delay per packet on various $\mathrm{CW}$ size on vehicular safety messages are CAM and DENM of the realistic highway model in ns-3.

The rest of the paper we described the related work, simulation scenario setup for different $\mathrm{CW}$, performance analysis, results and conclusion.

TABLE I: MODEL SPECIFIC PARAMETERS FOR A HIGHWAy [1], [7]

\begin{tabular}{cccccc}
\hline \hline Parameter & Unit & LOS & MD & SD & DI \\
\hline$G_{0}$ & $d B$ & -30 & $-100+24 n$ & $-100+24 n$ & 100 \\
$n$ & - & 1.6 & $\begin{array}{c}N((1.9,6.25, \\
0.35)\end{array}$ & $\begin{array}{c}N(1 ; 9: 61 ; \\
0 ; 3: 5)\end{array}$ & 8 \\
& & & & & \\
$\sigma_{a}^{2}$ & - & $\begin{array}{c}\operatorname{Exp}(6 . \\
8)\end{array}$ & $\operatorname{Exp}(9.4)$ & $\operatorname{Exp}(6.3)$ & - \\
\hline \hline
\end{tabular}




\begin{tabular}{cccccc}
\hline \hline$d_{\text {cmin }}$ & $m$ & 4.4 & 1.1 & 1.0 & - \\
$d_{\text {cerand }}$ & $m$ & $\begin{array}{c}\operatorname{Exp}(7 . \\
2)\end{array}$ & $\operatorname{Exp}(5.4)$ & $\operatorname{Exp}(4.9)$ & - \\
$\chi$ & $m^{-1}$ & & 0.005 & 0.005 & 1 \\
$W_{\text {road }}$ & $m$ & 17 & \\
$W_{\text {lane }}$ & $m$ & 4.25 & \\
$W_{D I}$ & $m$ & 5 & \\
$Y_{D I}$ & $m$ & 13.5 & \\
$y_{S D}$ & $m$ & $N(14,9)$ & \\
$d_{\text {ref }}$ & $m$ & $3 * 10^{\mathrm{9}} / 5.9 * 10^{9}$ \\
$\lambda$ & $m$ & 1 \\
$N_{R}$ & - & 1 \\
$N_{T}$ & - & 1 & \\
$N_{f}$ & - & 10 \\
$M$ & - & & \\
\hline \hline
\end{tabular}

\section{RELATED WORK}

In 2004 the IEEE 802.11 Task Group p (TGp) has been created for the wireless access vehicular network architecture and IEEE Standards Board approved the amendment proposed by TGp in June 2010 [7], [10]. In [1] investigate the data delivery performance at network layer and introduced a new channel model in ns-3 (e.g. Lund Model) based on the highway and rural environments in Lund city, Sweden. Lund propagation model is the wideband multiple-input-multiple-output (MIMO) model based on the MIMO channel measurements performed at 5.2 $\mathrm{GHz}$ band and introduced a generic geometry-based stochastic channel model (GSCM) approach and techniques in [7]. In [1] they have developed the upper network layer of Lund propagation loss model in ns-3 and integrate with lower physical layer using fixed CW size. Two-step Binary Exponential Backoff algorithm (TBEB) proposed by Zhu ea al. based on the two-dimensional Markov model used for the probability of the packet collision reduced, average CW size and number of backoffs [8].

TBEB is able to given better resetting its CW size which could obtained less oscillation then IEEE 802.11p adjustment. The minimum $\mathrm{CW}$ size adjustment depending on the vehicular density and improved the performance of IEEE 802.11 protocol [9]. Geographical-Location-Based Asynchronous Time Division Multiplexing (GLATDM) broadcast protocol can avoid the hidden terminal problem well, Chanel-State-Feedback-Based Back-off (CSFB) can assure for the reliable broadcast and Adaptive Dynamic Contention Window (ADCW) using for the adjust the CW size with changing the vehicles density [3], [10]. In [4] they propose a protocol alert message to overcome the delay sparse networks (82\% in dissemination delay) and rebroadcast messages in dense network (improvement up to $57 \%$ rebroadcast messages). The beacon reception probability of different CW size in broadcast IEEE $802.11 \mathrm{p}$ performance drops below $50 \%$ when the $33 \%$ exceeds the channel utilization [2]. The Dynamic Contention Window (DCW) proposed for the periodic broadcast scheme and shows the better performance in terms of the collision probability traditional fixed-CW-size-based broadcast in [11].

\section{IMPLEMENTATION OF VANET IN NS-3}

\section{A. About ns-3}

The discrete event-driven network simulator NS-3 is used for teaching and research area. It is being developed under GNU GPLv2 license and it is freely available for both user and development [12].

Some differences from the NS-2 include [12]:

- Replacement of Python into the OTcl in the simulation configuration.

- Simulation traces analysis in Wireshark.

- For study and research work it is easily integrating with real system in simulation.

- The system is well documented attribute.

\section{B. Equations}

The model developed by three types of point scatterers: mobile discrete (MD) scatterers represent other cars on the road. Static discrete (SD) scatterers denote buildings, farm houses, noise barrier for residential areas, embankments, road signs and other prominent obstacles. And finally, diffuse scatterers (DI) model the weak components that result mainly from the sides of the TX-RX path [1], [7]. These different types of scatterers are randomly distributed on a road strip in the $\mathrm{x}-\mathrm{y}$ plane. Furthermore, only the single bounces at the scatterers are assumed. Assuming a straight road along the $\mathrm{x}$-axis of width $\mathrm{W}$ _road, all scatterers $\mathrm{x}$ coordinates are uniformly distributed over $\left[x_{\min } ; x_{\max }\right]$. The y-coordinates of the MD scatterers follow a discrete uniform distribution, such that the possible y-values fall in the centre of a lane of width $W_{\text {lane }}$. The SD scatterer $y$ coordinates are Gaussian distributed, symmetrically around the road centre. The DI scatter points are placed in two uniform intervals with mean \pm y_DI and width $W_{D I}$, also symmetrically around the road center. The number of scatterers is dependent on the road length, i.e. for each type a density $\chi$ is specified.

The LOS and the discrete scatterer path amplitudes are modelled as slow fading, i.e. they vary over the distance because several paths are represented as a single one. All geometric calculations are done only in two dimensions in this model. The path contributions are given as,

$$
a(d)=g s \sqrt{G_{0}}\left(\frac{d_{r s f}}{d}\right)^{n_{1 / 2}}
$$

The amplitude contains a stochastic part through gs, which denotes correlated log-normal largescale fading, and a deterministic part. $G_{0}$ is the received power at a reference distance $d_{\text {ref }}$. The distance from transmitter to receiver via this particular scatterer with path loss exponent $n$ equals $d$. $g s$ is assumed to be stationary. The distance dependent auto covariance for $G_{g}=20 \log _{10} g s$ is given as,

$$
r_{d}(\Delta d)=E\left[G_{g} G_{g}(d+\Delta d)\right]=\sigma_{g}^{2} \exp \left(-\frac{\log 2}{d_{\varepsilon}^{2}}(\Delta d)^{2}\right)
$$


where the variance of the stochastic process is $\sigma_{g}^{2} . d_{c}$ is the coherence distance such that $\rho_{d}\left(d_{c}\right)=\frac{r_{d}\left(d_{c}\right)}{r_{d}(0)}=0.5$. Both $\sigma_{g}^{2}$ and $d_{c}$ follow exponential distributions, but the latter contains also a minimum value $>0$ such that $d_{c}=d_{\text {cmin }}+d_{\text {crand. }}$. For each scatterer individual values are assigned for the path loss exponent $n$, the coherence distance $d_{\varepsilon}$, the variance $\sigma_{\approx}^{2}$, the $x$ - and $y$-coordinate and for the MD scatterers the velocity $v$. The values for the constants and the distributions are listed in Table I. The amplitude of a diffuse scatterer does not fade with the distance. The path contribution is given as:

$$
a=\sqrt{\frac{G_{0}}{N_{D I}}\left(\frac{d_{r a f}}{d}\right)^{n_{d} / 2}}
$$

where, $N_{D I}$ equals the number of DI scatterers.

In the implementation a simpler version, a special case, has been used with a lossless isotropic antenna on transmitter, respectively receiver, which simplifies computational complexity. With respect to NS-3 integration, the final interesting quantity is the path loss. It is determined from the small-scale averaged channel gain $G_{g s a}$ according to [7] as:

$$
P L\left(t_{k}\right)=G_{a_{v} R}+G_{a_{2} T}-10 \log _{10} G_{g S a}\left(t_{k}\right)
$$

where,

$$
\begin{aligned}
& G_{g s a}\left(t_{k}\right)=\frac{1}{M N_{f}} \sum_{n_{R}=1}^{N_{R}} \sum_{n_{T}=1}^{N_{T}} \sum_{l=1}^{N_{f}} \sum_{m=0}^{M-1} \mid H\left[n_{R^{*}} n_{T^{*}} f_{i}, t_{k}+\right. \\
& m \Delta t]\left.\right|^{2}
\end{aligned}
$$

$G_{a}$ is the average antenna gain of receiver and transmitter $N_{f}$ denotes the number of sampled frequencies, $N_{R}$ and $N_{T}$ the number of receive and transmit antenna elements, $M$ ensures that the channel is stationary over the interval $M \Delta T$ and $H$ is the channel transfer matrix.

In our simulation, the main performance metrics are defined as follows:

$$
\begin{aligned}
& \text { Average delay per packet }=\frac{\text { Delay sum }}{R x \text { Packet }} \\
& \text { Total packet loss ratio }=\frac{\text { Last packets }}{R x \text { packets+ Lost packets }}
\end{aligned}
$$

\section{Simulation Specification}

This parameters and formulas listed here stem not just from the initial model specification in [7] but also from an (internal) MATLAB implementation from $\mathrm{K}^{\circ}$ aredal et al, which is optimized for speed. In our simulation, we used the Lund city, Sweden highway environment.

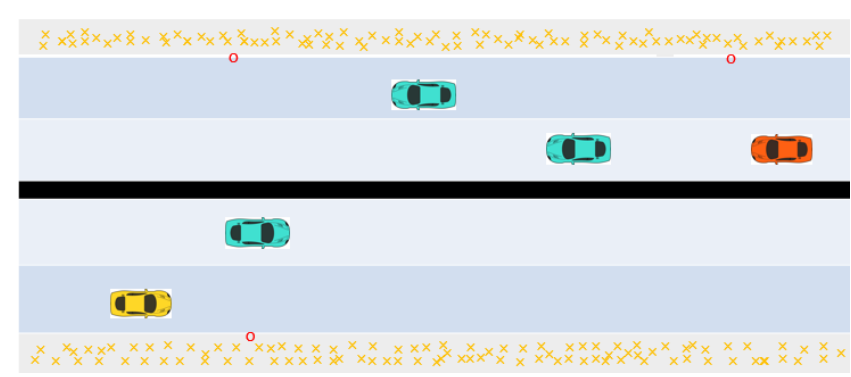

Fig. 1. Road strip and scatterer distribution for Highway.

In the Fig. 1 the brown (cross) represent as a diffuse scatterer, the static discrete are red (circle), the yellow car represents as a transmitter, blue car represents as a mdNodes, red car represents as a receiver and the symbols on the road denoted the starting positions of the cars. The line extending from those positions show how far the vehicles drive during the simulation run.

The scatterers distribution (position) of mdNodes in the Lund city highway the vilocity $v$, path loss exponent $n$, varience $\sigma_{g}^{2}$ of the stochastic amplitude gain and the coherence distance $d_{\text {croad }}$ are generated into the class LundModelGeometry. The transmitter and receiver are driving towards each other for $0.5 \mathrm{~s}$.

The default values in LundModelGeometry are a road strip of $500 \mathrm{~m}$ length, 4 lanes of width $4.25 \mathrm{~m}$ each and a total width of $17 \mathrm{~m}$. Have a look in the different classes or the doxygen documentation to find out more about the attribute names.

All mdNodes (cars) even those that do not have a WiFi interface and serve only as mobile discrete scatter points. Lund city highway model can help with the random distribution of cars. NodeContainer for $T x$ and $R x$ nodes can be created. Individual car speeds (mdNodes) were randomly assigned according to a normal (Gaussian) distribution with the parameters of, Mean Speed $=25 \mathrm{~m} / \mathrm{s}$ and Speed Variance $=4.0 \mathrm{~m} / \mathrm{s}$.

The parameters are passed on to LundModelGeometry to generate the scatter points and to LundModelLsFading to calculate the large-scale fading for each TX-RX pair. The latter is determined for a simulation duration tMax and transmissions with period packetInterval. The variables txNodes, rxNodes and mdNodes are of type NodeContainer, tMax and packetInterval of type double. Simulations were run with a bash script for batch execution with different fixed environments and random number generator seeds, respectively run numbers.

\section{RESULTS}

We have presented the effect of different $\mathrm{CW}$ size in a realistic highway scenario based on the Lund city model specification [1,7]. According to the vehicular safety and improve the traffic efficiency vehicular are communicate by using two main message models are Cooperative Awareness Message (CAM) and Decentralized Event Notification Message (DENM). Neighboring vehicles are communicating CAM message. This message contains vehicle identification, speed, geographical location and the basic status of neighboring vehicles. The DENM used for hazardous events (e.g. accidents) has taken place on the road and broadcast emergency message for rescue. In our simulation we have used the following specification, 
TABLE II: SIMULATION SPECIFICATION [12].

\begin{tabular}{ll}
\hline \multicolumn{1}{c}{ Attribute Name } & \multicolumn{1}{c}{ Attribute Value } \\
\hline WAVE operating frequency & $5 \mathrm{GHz}$ \\
WAVE Data Rate & $6 \mathrm{Mbps}$ \\
WAVE Bandwidth & $10 \mathrm{MHz}$ \\
Packet size & $\mathrm{CAM} \mathrm{(200} \mathrm{Bytes);} \mathrm{DENM} \mathrm{(100} \mathrm{Bytes)}$ \\
Frequency of the packet & $\mathrm{CAM} \mathrm{(10} \mathrm{Hz);DENM} \mathrm{(20} \mathrm{Hz)}$ \\
Highway street length & $500 \mathrm{~m}$ \\
\hline \hline
\end{tabular}

In our simulation we simulated different $\mathrm{CW}$ size of three ns-3 propagation loss models (Three log distance, Friis model, Nakagami model) into the Lund city highway environment by using CAM and DENM messages.

For CAM message Nakagami propagation model shows the maximum average delay per packet between $\mathrm{CW} 128$ and $\mathrm{CW}$ 512. But in $\mathrm{CW} 64$ the three log distance model was more than 50 milliseconds where Friis model shows around 45 milliseconds. Three log distance model shows the maximum packet loss ratio for different $\mathrm{CW}$ size.

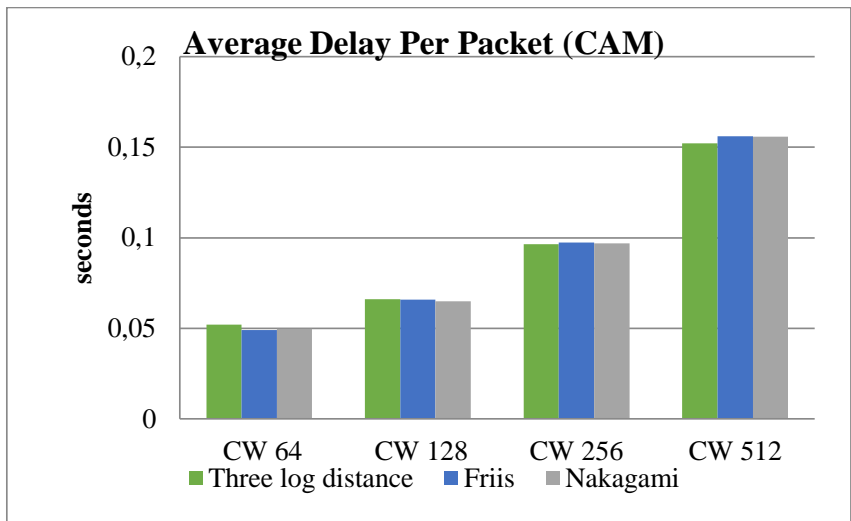

Fig. 2. Average delay per packet (CAM)

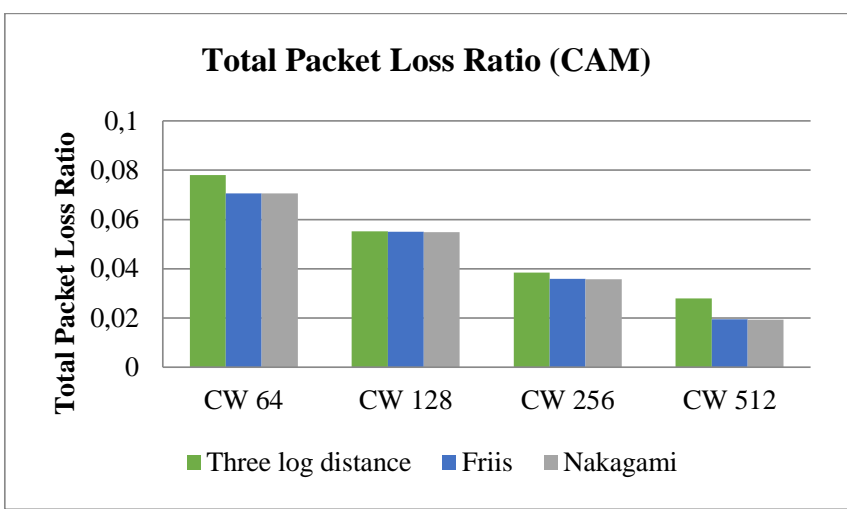

Fig. 3. Total packet loss ratio (CAM)

However, for DENM messages the average delay per packet for Nakagami model and Friis model are nearly same, but three log distance model gives the minimum delay per packet on different $\mathrm{CW}$ size. Total packet loss ration diminished by increasing $\mathrm{CW}$ size. Friis model gives the minimum packet loss ratio (around 24\%).

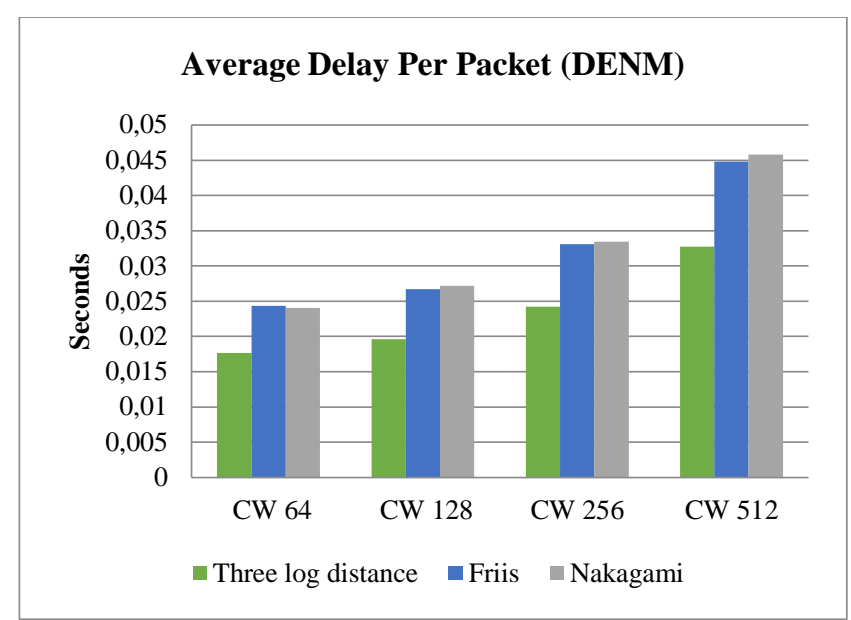

Fig. 4. Average delay per packet (DENM)

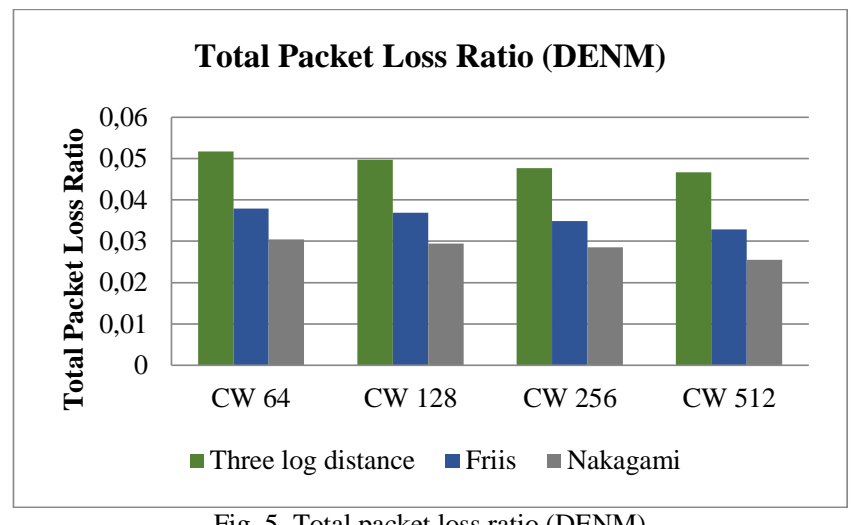

Fig. 5. Total packet loss ratio (DENM)

\section{CONCLUSION}

In this paper, we simulated a realistic highway model in ns- 3 based on high resolution algorithm parameters and the measurement environment. We create a realistic two-lane highway road environment and simulated it in ns-3 by using different $\mathrm{CW}$ size, vehicular safety messages and three default existing ns-3 propagation loss models.

Average delay per packet has been increase by raising the CW size for both CAM and DENM messages. Three log distance model gives the minimum average delay per packet. By increasing the $\mathrm{CW}$ size the total packet loss ratio decreased and Nakagami model gives the minimum packet loss ratio.

\section{REFERENCES}

[1]. G. Eason, B. Noble, and I.N. Sneddon, "On certain integrals of Lipschitz-Hankel type involving products of Bessel functions," Phil. Trans. Roy. Soc. London, vol. A247, pp. 529-551, April 1955.

[2]. R. Reinders, M. van Eenennaam, G. Karagiannis and G. Heijenk, "Contention window analysis for beaconing in VANETs," 2011 7th International Wireless Communications and Mobile Computing Conference, Istanbul, 2011, pp. 1481-1487.

[3]. S. Wang, B. Yang, F. Gu and X. Shi, "A novel reliable broadcast protocol for VANET's safety applications," 2016 6th International Conference on Electronics Information and Emergency Communication (ICEIEC), Beijing, 2016, pp. 282-286.

[4]. A. Berradj and Z. Mammeri, "Adaptive Contention Window Scheme to Improve Multi-Hop Broadcast in VANETs," 2015 IEEE 81st Vehicular Technology Conference (VTC Spring), Glasgow, 2015, pp. 1-6.

[5]. D. Rossi, R. Fracchia and M. Meo, "VANETs: Why Use Beaconing at All? "2008 IEEE International Conference on Communications, Beijing, 2008, pp. 2745-2751. 
[6]. Y. Lu, J. Ren, J. Qian, M. Han, Y. Huo and T. Jing, "Predictive Contention Window-Based Broadcast Collision Mitigation Strategy for VANET," 2016 IEEE International Conferences on Big Data and Cloud Computing (BDCloud), Social Computing and Networking (SocialCom), Sustainable Computing and Communications (SustainCom) (BDCloud-SocialCom-SustainCom), Atlanta, GA, 2016 , pp. 209-215.

[7]. J. Karedal et al., "A geometry-based stochastic MIMO model for vehicle-to-vehicle communications," in IEEE Transactions on Wireless Communications, vol. 8, no. 7, pp. 3646-3657, July 2009.

[8]. Y.-H. Zhu, H. Xu, and J. Peng, "Two-step backoff algorithm for ieee 802. 11 based wireless networks," Dianzi Yu Xinxi Xuebao(Journal of Electronics and Information Technology), vol. 33, no. 11, pp. 25752581, 2011.

[9]. R. Stanica, E. Chaput and A. L. Beylot, "Local density estimation for contention window adaptation in vehicular networks," 2011 IEEE 22nd International Symposium on Personal, Indoor and Mobile Radio Communications, Toronto, ON, 2011, pp. 730-734.

[10]. IEEE Standard for Information Technology Telecommunications and Information Exchange between Systems - Local and Metropolitan Area Networks - Specific Requirements - Part 11: Wireless LAN Medium Access Control (MAC) and Physical Layer (PHY) Specifications - Amendment 6: Wireless Access in Vehicular Environments, July 2010.
[11]. Q. Yang, S. Xing, W. Xia and L. Shen, "Modelling and performance analysis of dynamic contention window scheme for periodic broadcast in vehicular ad hoc networks," in IET Communications, vol. 9, no. 11, pp. 1347-1354, 7232015.

[12]. Islam Md Ashraful, Bhuyan Nasimul Hyder Maruf, "The Effect of Radio Channel Modelling on the Network Performance in VANET" Department of Electrical and Information Technology Faculty of Engineering, LTH, Lund University SE-221 00 Lund, Sweden, 2015.

Nasimul Hyder Maruf Bhuyan completed MSc in Radio Communication from Blekinge Tekniska Högskola (BTH), Sweden. He completed his master's thesis from Lund University, Sweden. Now, he is working on sensor network and 5G communication. His research interests are on wireless sensor networks, network security and wireless communication.

Sabrina Alam received B.Sc. degree and M.S. degree in Applied Physics, Electronics and Communication Engineering from University of Chittagong, Bangladesh in 2009 and 2010 respectively. Her current research interests are Wireless Communications, Optical Switching and Optical Fiber Communications, Solid state Electronic devices. She is currently working as an assistant professor in the Department of Electrical and Electronic Engineering, University of Chittagong, Chittagong-4331, Bangladesh. 